\title{
The Formulation Design and Experimental Research on Damping Performance Optimization of Polymer Blends Materials
}

\author{
Chao Qian ${ }^{1, a}$, Zhimin Chen ${ }^{1 *}$,, Yongqing $\mathrm{Li}^{2, \mathrm{c}}$ \\ ${ }^{1}$ Office of Research \& Development, Naval University of Engineering, Wuhan, 430033, China \\ ${ }^{2}$ Warship Engineering Department, Naval University of Engineering, Wuhan, 430033, China \\ aemail: bluesea0520@163.com, bemail:czm12345678@yeah.net
}

Keywords: Dynamic Mechanical Property; Polymer Blends (PB); Formulation Design

\begin{abstract}
Because polymer blends(PB) performance is affected by its components and contents, so one of the most important research goals is the creation of an optimal design of the polymer blends formulation. However, the traditional orthogonal experiment was difficult and inefficient,due to the fact that polymer blends has many components and varying contents. Throughout this paper, a combination of uniform design and multiple regression statistical analyses were employed to optimize the formulation of polymer blends. Our methods were verified by experiments. The results showed a consistency with our hypothesis, and the workload was reduced. It means that the experimental method is an effective strategy for optimizing polymer blends formulations.
\end{abstract}

\section{Introduction}

There have been many formulation optimization methods used in the process of the synthesis of polymer blends. Traditionally, the components' optimization design has been based principally onsynthesis mechanisms, which include physicochemical properties and the interactions between components. After the blended components are determined, the ratios between each component are the main factors influencing the properties of the polymer blends. So, the component optimization of multicomponent materials must be further studied. The common optimization strategies are mainly based on experimental methods, including the orthogonal design method, the uniform experiment, and some other means. When compared with other methods, the uniform experiment requires lesser samples and provides an easier regression analysis. Furthermore, it is a helpful method in multicomponent optimization processes.

This paper determined the multicomponent relative ratio of the given polymerblends material by the optimization of different component ratios of high damping materials. In addition, we established the ratio equation of state function, allowing for the best relative ratio to be found. Finally, experimental verification and comparative analysis were studied through synthesis experiments and damping performance tests.

\section{The basic theory}

\section{Uniform design.}

Uniform design is a new method of experimental design that combines number theory and multivariate statistical analysis. Unlike orthogonal design, uniform design does not need to consider the neat comparability of data, but only deals with the uniform dispersion of experimental points in a limited range. That is to say, the data system features may be studied effectively by choosing lesser experimental points from the whole data set. The main characteristics of the uniform design are: a) investigation of each of the factors using only one experiment at each level; b) any two factors on the lattice points in the plane having only one experimental point on each row and each column; c) the increase of experiments being consistent with the augmentation of numbers of levels.

In uniform design, each of these factors use only one experiment at each level, so the number of experiments is equal to the number of levels. Thus, the workload is greatly reduced as compared 
with orthogonal experiments. In uniform design, the experiments are arranged by a series of welldesigned tables. In order to get the highest level of uniformity, each table is accompanied by a set of instructions to select the appropriate column from the uniform design table.

\section{Optimization design method of formulation experiment.}

Formulation design of polymer materials is a traditional kind of diversity and complexity experimental design with multiple factors, levels, and indexes. So it is workload, high-cost, and long-cycle by choosing an orthogonal experiment as a formulation optimization method. Uniform design is a new kind of design method that combines number theory and multivariate statistics. Applying uniform design to experiments with multiple factors, levels, and indexes effectively reduces the number of experimetns. At the same time, multiple linear regression can be used as a method of data analysis, allowing small amounts of experimental results to yield more information. It also simplifies the experimental process and improves its effiency[1]

Orthogonal designs can make it very intuitive to analyze how factors and levels affect the changing of the index. However, in a uniform design, there is a need for data processing. During uniform design, many data processing methods, like multiple regressions, are used to establish regression equations between performance parameters and variables. In practicality, if there is more than one factor that influence the variable in a regression problem, then this kind of regression is named as multiple regression[2]. More information can be obtained from a regression equation, such as: a) the establishment of a quantitative relationship between variables by one set of data; b) the performance of statistical tests to verify the credibility of relationship between variables; c) the determination of which variables are more important to a certain factor; d) the prediction and control of the experiment by using the relationship between variables.

\section{Experiment and Characterization}

\section{Experiment.}

The polymer blends composed of polyurethane(PU) / epoxy(EP) / unsaturated polyester resin(UPR) were synthetized.The polyurethane pre-polymer (PPU) with a -NCO termination was synthetized by a reaction between pretreatment polyether and TDI with a mole ratio of 1:2. According to reference[3], a series of PPU based polymer blendss were synthetized. The epoxy resin was the diglycidyl ether of bisphen of A (DGEBA), which was generously provided by Yueyang Petrochemical Co. China.

\section{Properties test.}

The damping performance of PB was characterized by the damping loss factor(tan $\delta)$ and the specific damping temperature range $\Delta \mathrm{T}(\tan \delta>0.3)$. The dynamic mechanical property was studied by a Japanese-made DDV- II dynamic viscoelastic spectrometer, with a testing frequency of $3.5 \mathrm{~Hz}$, a heating rate of $2^{\circ} \mathrm{C} / \mathrm{min}$, and a temperature range of $-150{ }^{\circ} \mathrm{C} \sim 200^{\circ} \mathrm{C}$. The samples were cast in film with the size of $60 \mathrm{~mm} \times 4 \mathrm{~mm} \times 0.4 \mathrm{~mm}$.

\section{$\mathrm{PB}$ ratio optimization and experimental results}

The damping performances of PU/EP/UPR are characterized by the dynamic mechanical thermal property.The damping loss factor $(\tan \delta)$ and the specific damping temperature range $(\Delta \mathrm{T})$ are the two most important factors of damping performance. In this uniform design, $\tan \delta$ and $\Delta \mathrm{T}$ are subjective functions (marked as $\mathrm{y}_{1}$ and $\mathrm{y}_{2}$ ), while the mass ratio between PU, EP, and PUR are regarded as variables, and the number of the component variation factor (marked as $S$ ) is 3 . As shown in Table 1, according to reference[4], Uniform Table $\mathrm{U}_{5}\left(5^{4}\right)$ is chosen as the uniform table, and Table 2 serves as the instruction table. 
Table.1 Uniform Table $\mathrm{U}_{5}\left(5^{4}\right)$

\begin{tabular}{|c|c|c|c|c|}
\hline Experiments\Column & 1 & 2 & 3 & 4 \\
\hline 1 & 1 & 2 & 3 & 4 \\
\hline 2 & 2 & 4 & 1 & 3 \\
\hline 3 & 3 & 1 & 4 & 2 \\
\hline 4 & 4 & 2 & 3 & 1 \\
\hline 5 & 5 & 5 & 5 & 5 \\
\hline
\end{tabular}

Table.2 Theinstruction tableof Table1

\begin{tabular}{|c|c|}
\hline Factors & Column \\
\hline 2 & 1,2 \\
\hline 3 & $1,2,4$ \\
\hline
\end{tabular}

According to Table2, columns 1, 2and 4 in Table1 should be chosen as the performance test data, because $S=3$. The formulation design and the results are presented in Table3.

Table.3 Formulation design and the results of PU/EP/UPR system

\begin{tabular}{|c|c|c|c|c|c|}
\hline \multirow{2}{*}{ Experiments } & \multicolumn{2}{|l|}{ Factors/(mass ratio of each component) } & \multicolumn{2}{|c|}{ Results } \\
\cline { 2 - 6 } & $\begin{array}{c}\mathrm{w}_{1} \\
\text { (PPU) }\end{array}$ & $\mathrm{w}_{2}$ (EP) & $\mathrm{w}_{3}$ (UPR) & $\mathrm{y}_{1}$ & $\mathrm{y}_{2}$ \\
\hline K01 & $1(4)$ & $2(8)$ & $4(16)$ & 0.72 & 73 \\
\hline K02 & $2(8)$ & $4(16)$ & $3(12)$ & 0.52 & 88 \\
\hline K03 & $3(15)$ & $1(5)$ & $2(10)$ & 0.8 & 67 \\
\hline K04 & $4(16)$ & $3(12)$ & $1(4)$ & 0.4 & 58 \\
\hline K05 & $5(10)$ & $5(10)$ & $5(10)$ & 0.61 & 68 \\
\hline
\end{tabular}

First, the performance test data should be processed by multiple regression. Then, the multiple regression equations between the mass ratio and $\tan \delta$, as well as that between the mass ratio and $\Delta \mathrm{T}$ are established. As shown in Equations(1)and(2), $\mathrm{w}_{1}, \mathrm{w}_{2}$ and $\mathrm{w}_{3}$ refer to the relative quality of PU, $\mathrm{EP}$, and UPR, respectively.

\section{1) Multiple regression equation between mass ratio and $\tan \delta$}

$$
y_{1}=a_{0}+a_{1} w_{1}+a_{2} w_{2}+a_{3} w_{3}=-0.033+0.0282 \mathrm{w}_{1}-0.017 \mathrm{w}_{2}+0.049 \mathrm{w}_{3}
$$

According to Equation (1) $a_{0}, a_{1}, a_{2}$, and $a_{3}$ are equal to $-0.033,0.0282,-0.017$, and 0.049 respectively, while the standard deviationsare $0.352,0.013,0.007$,and 0.015 , respectively. The standard deviation of $y_{1}$ is 0.041 , while thestandard correlation coefficientbetween $y_{1}$ and $w_{1}, w_{2}$, $w_{3}$ is 0.99 . It can be noticed that $w_{1}$ and $w_{3}$ have positive coefficients, signaling the increase of tan $\delta$ with increasing PU and UPR content.

\section{2) Multiple regression equation between mass ratio and $\Delta \mathbf{T}$}

$$
y_{2}=b_{0}+b_{1} w_{1}+b_{2} w_{2}+b_{3} w_{3}=-14.67+3.01 w_{1}+2.875 w_{2}+5.22 w_{3}
$$

According to Equation (2), $b_{0}, b_{1}, b_{2}$, and $b_{3}$ are equal to $-14.67,3.01,2.875$, and 5.22 respectively, while the standard deviations are $7.59,0.66,0.36$, and 0.76 , respectively. The standard deviation of $y_{2}$ is 2.04, and the standard correlation coefficient of $y_{2}$ and $w_{1}, w_{2}$, and $w_{3}$ is 0.99 . It can be noticed that $w_{3}$ has a larger positive coefficient, meaning that in a PB system, $\Delta \mathrm{T}$ increases as UPR content increases.

After establishing the regression equation, based on the range of the formulation variety, the value of the objective function can be calculated by the exhaustive method[5] [6]. The large amount of calculation allows this process to be completed by a computer program. For example, in order to get a PB material with a higher $\tan \delta$ peak and a broader $\Delta \mathrm{T}$, we input a satisfactory value of $\tan \delta$ and $\Delta \mathrm{T}\left(\mathrm{y}_{1}>0.6, \mathrm{y}_{2}>60\right)$ into the computer and the corresponding formulation and performance factors are given by computer programming. The optimization formulation and the performance parameters of tertiary polymer blends are displayed in Table 4. 
Table.4 The optimization formulation and performance of ternary polymer blends

\begin{tabular}{|c|c|c|c|c|c|}
\hline \multirow{2}{*}{ Formulation } & \multicolumn{3}{|c|}{ Component (w\%) } & \multicolumn{2}{c|}{ Optimization results } \\
\cline { 2 - 6 } & $\mathrm{w}_{1}(\mathrm{PPU})$ & $\mathrm{w}_{2}$ & $\begin{array}{c}\mathrm{w}_{3} \\
(\mathrm{EP})\end{array}$ & $\begin{array}{c}\mathrm{y}_{1} \\
(\tan \delta)\end{array}$ & $\begin{array}{c}\mathrm{y}_{2} \\
(\Delta \mathrm{T})\end{array}$ \\
\hline 1 & 15.42 & 5.73 & 7.85 & 0.69 & 89.2 \\
\hline 2 & 14.36 & 5.61 & 9.04 & 0.72 & 91.9 \\
\hline
\end{tabular}

According to the optimization results, the PB samples, denoted by I01 and I02, are prepared by Formulations 1 and 2 respectively. The dynamic mechanical thermal properties of I01 and I02 are shown in Figure1 and Figure2.

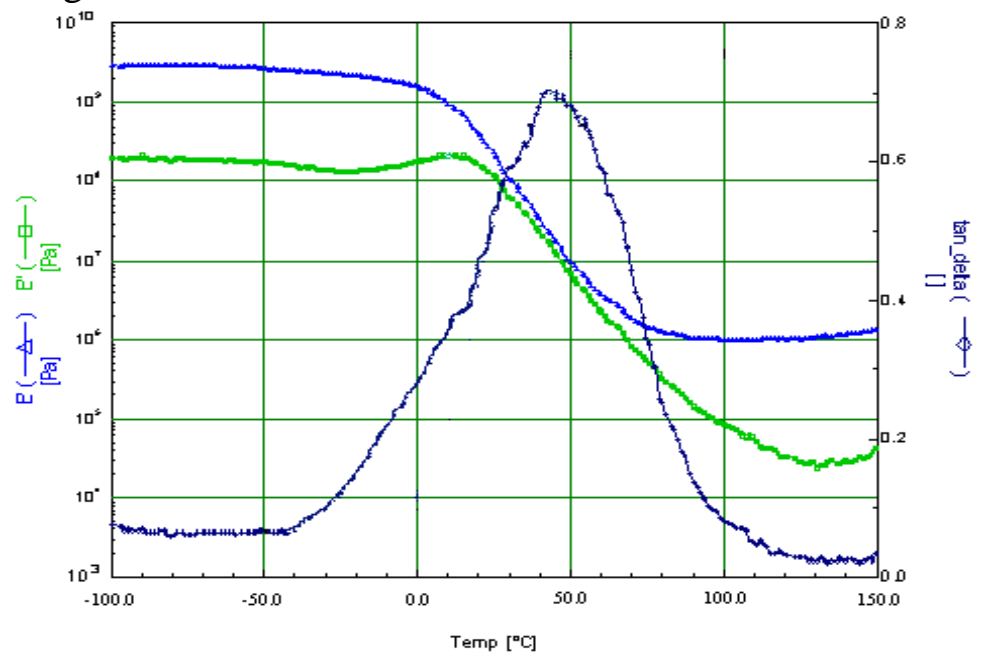

Fig. 1 The DMTA of I01

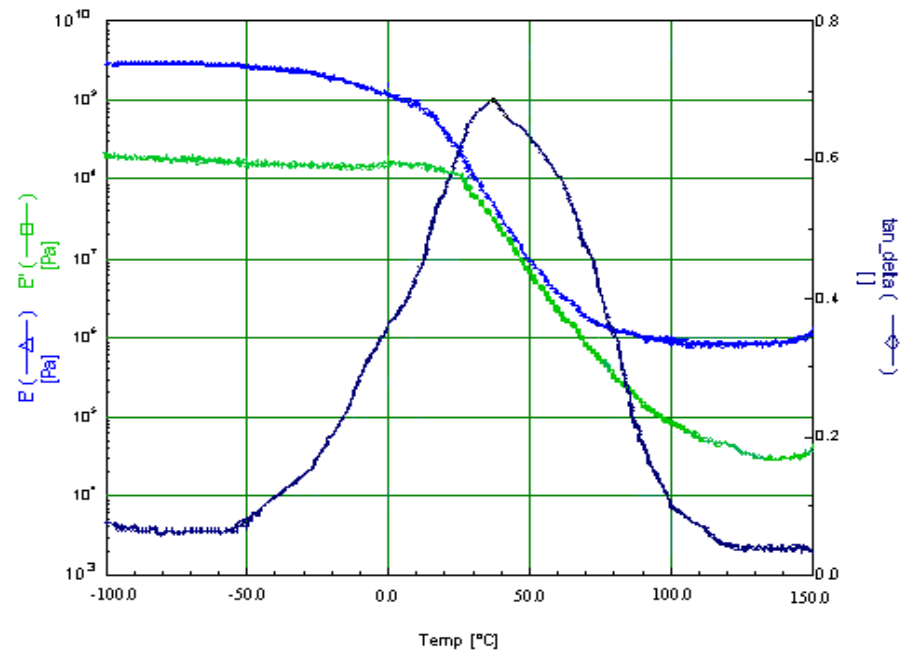

Fig.2 The DMTA of I02

According to Figure1 and Figure2, we discover that the calculated value is close to the actual value, and that $\Delta \mathrm{T}$ is broadened. It can be seen that a uniform design is well-used in the formulation optimization of tertiary polymer blends and contributesto the synthesizing of novel damping materials[7] [8].

\section{Conclusion}

Uniform design lessens the experimental workload and demonstrates the important factors affecting the dynamic mechanical thermal properties of polymer blends. It is an effective method for formulation optimization of multicomponent polymeric system. The combination of uniform design and multiple regression analysis is used to optimize the formulation of polymer blends. The $\tan \delta$ and $\Delta \mathrm{T}(\tan \delta>0.3)$ values, calculated by this new method,are close to the values obtained when 
testing the method by dynamic mechanical thermal analysis. This illustrates that our method can be used to formulate the optimization of multicomponent polymer blends.

\section{References}

[1] T.R. Zhang, Z.X Ma, Q.Z Yang. Modern Rubber Formulation[M]. Beijing: Chemical Industry Press, 1996.

[2] K.T. Fang. Practical Regression Analysis[M]. Beijing: Science Press, 1986.

[3] Y.Q. Li. Based on Interpenetrating Polymer Network of Underwater Acoustic Sound-absorbing Composite Materials[D]. Naval University of Engineering, 2008: 28-35.

[4] K.T. Fang. Uniform Design and Uniform Design Table[M]. Beijing: Science Press, 1994.

[5] L. XU, X.G. Shao. Methods of Chemometrics[M]. Beijing: Science Press, 2004.

[6] Fletcher R. Practical Optimization Method(The second edition) [M]. New York: Wiley, 1987.

[7] L.C. Qin, J. Cai, J.S. Zhang, et al. Structure and Properties of Interpenetration Polymer Networks Materials with Wide-temperature Range and Damping Properties[J]. China Synthetic Rubber Industry, 2004, 27(6): 374-378.

[8] B.C. Guo, Q.H. Qiu, D.M. Jia. Mechanical Properties and Morphologies of Conjugated Threecomponent Interpenetrating Polymer Networks - Polyurethane/Poly(methyl methacrylate)/Scrap Rubber System[J]. Journal of South China University of Technology(Natural Science Edition), 2000, 28(3): 10-16. 